\title{
Design of 2D Time-Varying Vector Fields
}

\author{
Guoning Chen, Member, IEEE, Vivek Kwatra, Li-Yi Wei, \\ Charles D. Hansen, Fellow, IEEE, and Eugene Zhang, Senior Member, IEEE
}

\begin{abstract}
Design of time-varying vector fields, i.e., vector fields that can change over time, has a wide variety of important applications in computer graphics. Existing vector field design techniques do not address time-varying vector fields. In this paper, we present a framework for the design of time-varying vector fields, both for planar domains as well as manifold surfaces. Our system supports the creation and modification of various time-varying vector fields with desired spatial and temporal characteristics through several design metaphors, including streamlines, pathlines, singularity paths, and bifurcations. These design metaphors are integrated into an element-based design to generate the time-varying vector fields via a sequence of basis field summations or spatial constrained optimizations at the sampled times. The key-frame design and field deformation are also introduced to support other user design scenarios. Accordingly, a spatial-temporal constrained optimization and the time-varying transformation are employed to generate the desired fields for these two design scenarios, respectively. We apply the time-varying vector fields generated using our design system to a number of important computer graphics applications that require controllable dynamic effects, such as evolving surface appearance, dynamic scene design, steerable crowd movement, and painterly animation. Many of these are difficult or impossible to achieve via prior simulation-based methods. In these applications, the time-varying vector fields have been applied as either orientation fields or advection fields to control the instantaneous appearance or evolving trajectories of the dynamic effects.
\end{abstract}

Index Terms-Time-varying vector fields, 2D vector fields, vector field design, dynamic effects for surfaces.

\section{INTRODUCTION}

$\mathrm{V}$ ECTOR field design is a fundamental component for a variety of graphics applications such as remeshing [1], [33], texturing [20], [13], [23], [31], [41], [46], and nonphotorealistic rendering [16], [17]. The paramount importance of vector fields in these applications has invoked a line of comprehensive study on the techniques of vector field design on surfaces [6], [8], [34], [51]. Nonetheless, prior research has paid little attention to the more natural and general applications of vector field design to modeling dynamic effects, such as fluid animation [35], [36], crowds [4], [29], shape deformation [45], and video editing [18]. This is partly due to the fact that such dynamic systems are usually time varying (or time dependent), with the additional time dimension significantly increasing the complexity of the possible dynamics in the vector fields. In addition, there is no existing theory for the characterization of time-varying vector fields, compared to the welldefined feature characterization of static vector fields upon which the design techniques are built. For the first time, this

- G. Chen and C.D. Hansen are with the Scientific Computing and Imaging Institute, University of Utah, 72 S Central Campus Drive, Room 3750, Salt Lake City, UT 84112. E-mail: \{chengu, hansen\}@sci.utah.edu.

- V. Kwatra is with the Google Inc., 1600 Amphitheatre Parkway, Mountain View, CA 94043. E-mail: kwatra@gmail.com.

- L.-Y. Wei is with the Department of Computer Science, the University of Hong Kong, Room 301, Chow Yei Ching Building, Pokfulam Road, Hong Kong and the Microsoft Research, Redmond, WA 98052-6399.

E-mail: liyiwei@stanfordalumni.org.

- E. Zhang is with the School of Electrical Engineering and Computer Science, Oregon State University, 2111 Kelley Engineering Center, Corvallis, OR 97331. E-mail: zhange@eecs.oregonstate.edu.

Manuscript received 12 Feb. 2011; revised 30 Nov. 2011; accepted 14 Dec. 2011; published online 21 Dec. 2011.

Recommended for acceptance by S. Takahashi.

For information on obtaining reprints of this article, please send e-mail to: tvcg@computer.org, and reference IEEECS Log Number TVCG-2011-02-0032.

Digital Object Identifier no. 10.1109/TVCG.2011.290. paper systematically studies the design of time-varying vector fields on $2 \mathrm{D}$ manifolds, including the applications and the taxonomy of the vector fields, the requirements, and the appropriate techniques.

\subsection{Requirements}

For most graphics applications involving dynamic effects, there are a number of requirements for the underlying timevarying vector fields and how they are modeled.

First, the obtained time-varying vector fields should preserve temporal coherence to guarantee the smooth transition of the dynamic effects that they are driving. This is a fundamental requirement for achieving a visually pleasing animation.

Second, the obtained time-varying vector fields can be physically plausible or implausible, incompressible or compressible, in order to satisfy the requirements of different applications. For instance, practitioners in fluid dynamics often require incompressible flows, while animators may seek for more flexible vector fields for the dynamic effects with volume change such as crowd simulation. Any vector field system needs to be able to handle general timevarying vector fields with similarly diverse properties.

Third, the time-varying vector fields are designed to either control the evolution of the instantaneous appearance of certain graphical primitives (e.g., the sizes and orientations of the texture and brush strokes) or advect certain objects (e.g., flow parcels) over time, in order to control different aspects of the dynamic effects. A vector field design system should facilitate the creation of the vector fields for both types of use.

Fourth, the design system for the time-varying vector fields should provide the user an intuitive and flexible interface to support the modeling of various flow behaviors. In addition, a number of different modeling approaches 
should be supported. Specifically, there are a few possible situations during the modeling of a time-varying vector field that a user may encounter: 1) The user wishes to design the detailed local behavior of the flow over time; 2) The user cares about the exact states of the flow at only certain times and would like the system to generate the rest of the field; and 3) The user is given a static vector field, and tries to deform it to make up a time-varying vector field as people do for mesh deformation. A properly devised design system should be able to accommodate these scenarios.

\subsection{Our Method}

In order to develop a design system that satisfies the aforementioned requirements, we propose a design framework that is built on the discretization of the time-varying vector fields in the time dimension such that they can be considered as the sequences of static vector fields with slow changes over time. This philosophy is based on an observation that solutions to the time-varying vector fields converge to families of solutions of the instantaneous vector fields as the rate of temporal change in the vector field goes to zero, which preserves temporal coherence and helps achieve smooth transition of the dynamic effects. This observation is also a fundamental assumption when developing bifurcation theory for time-varying vector fields [11]. With this temporal discretization, we are able to adapt the previously developed tools for static vector field design to time-varying vector fields with the desired instantaneous dynamics.

To enable the creation of various flows, we provide the user with the ability of modeling the following flow properties:

1. a snapshot of the flow at a given time;

2. the path of a particle in the domain;

3 . the path of a singular feature; and

4. the interaction of the features of interest.

These features in turn reflect important flow characteristics, such as the solution of the dynamical system at a given time, the trajectories of the flow parcels, and how the flow parcels interact over time. These flow characteristics can be described by streamlines, pathlines, singularity paths, and bifurcations, respectively. They sufficiently describe the local flow behavior in space and time, and thus can be used to create time-varying vector fields for aligning or advecting graphical primitives as required. We refer to vector fields that are used for orienting graphical primitives as orientation fields and advecting objects as advection fields. We provide the design metaphors for the user to model these flow characteristics. Particularly, we present the first technique that allows the user to prescribe bifurcations, a unique type of phenomena not present in static fields.

To support the required design scenarios, we introduce three distinct field design approaches. Specifically, the modeling of the local flow behaviors is supported by the time-varying design elements extracted from the user-specified flow characteristics. A basis field summation or a constrained optimization is performed to generate the instantaneous vector field at a given time, based on the instantaneous characteristics of the elements. Key-frame design is employed to support the case when a user only provides the instantaneous fields at the desired times. A spatial-temporal Laplacian relaxation is proposed to generate the rest of the sequence. Time-varying transformation is used when an initial static field is deformed over time to produce a time-varying vector field.

The combination of the proposed design metaphors and generation techniques has led to a design system which takes the user input and generates a time-varying vector field using one of the generation approaches according to the selected design approach. The system also enables the user to further modify the obtained vector field through local topological editing. The generated time-varying vector fields can be applied to a number of important computer graphics applications to achieve various dynamic effects including producing artistic fluid effects over static images, steering $2 \mathrm{D}$ crowds, and controlling various time-varying effects on surfaces.

\section{Related Work}

Vector field design refers to the creation of a continuous vector field on a manifold that respects user-specified or application-dependent constraints. Most existing work focuses on a static vector field. Depending on the goals, there are two different classes of vector field design techniques: one is nontopological based; the other is topological based.

Nontopological-based methods. Nontopological-based methods do not address vector field topology [15] explicitly. The vector field design tools in the early graphics applications, such as texture synthesis [41], [46], fluid simulation [35], [36], and visualization [43], are examples of this category. Other applications, such as nonphotorealistic rendering [16], [17], remeshing [1], and parameterization [33], also employ vector field design, respectively. Most of these applications require only the direction information of the input vector fields, and hence a simple design functionality. However, the user has little control of unwanted singularities in the field that often lead to visual artifacts.

Topological-based methods. Topological-based approaches allow the user to control the number and positions of singularities [44], [51], [8] or the topological graph explicitly [37]. General N-way rotational symmetry field design has also been studied by Palacios and Zhang [27], Ray et al. [34], and Lai et al. [21]. Recently, Crane et al. [6] present a technique which allows arbitrary prescription of singularities and constraints on the fields.

Time-varying methods. Most of the above work concerns only time independent (i.e., static) vector fields. On the other hand, many applications are driven by timevarying vector fields, such as fluid simulation [35], crowd animation [39], [29], shape deformation [45], hair modeling [10], and video editing [50]. However, there is no interface that allows the user to intervene the underlying timevarying vector fields. This has restricted the achievable effects. Wejchert and Haumann [47] introduce the idea of flow design to create controllable aerodynamics animation. The modeled field is steady and needs to be combined with physically based simulation to generate aerodynamics animation. To achieve time-dependent control, the user exerts external force to the system as demonstrated by Stam [35], [36]. However, simulation is expensive and hard to 
control. In addition, simulation is incapable of generating physically impossible artistic fluid flow effects. Pighin et al. [30] introduce an interactive pathline editing interface and an advected radial basis function to model and edit incompressible flows. Compared to their work, our techniques enable the user to create $2 \mathrm{D}$ vector fields with more general characteristics than incompressible flows. Kagaya et al. [18] present a design interface to control time-varying tensor fields for the temporarily coherent painterly rendering of videos. $\mathrm{Xu}$ et al. [49] describe a technique for fast generation of static vector fields to assist interactive design. Ma et al. [24] propose a motion field synthesis technique that enables the user to generate artistic flow effects. However, the method only generates detailed motion vectors and relies on a predetermined low-resolution dynamic vector field for synthesis. To that end, we are not aware of any work on the design of time-varying vector fields for the general purpose of graphics applications.

\section{Overview}

In this section, we provide a brief description of how our framework assists the design of a time-varying vector field. First, the user specifies the desired flow characteristics using the following flow descriptors:

\begin{tabular}{l}
\hline \multicolumn{1}{|c|}{ flow descriptors } \\
of the flow geometry at a \\
certain time frame and most \\
useful for the design of ori- \\
entation fields \\
Pathline, for the descrip- \\
tion of movements of spe- \\
cific particles across space \\
and time (appropriate for ad- \\
vection fields) \\
Singularity path, for the \\
representation of the trajec- \\
tory of the singular features \\
over space and time (useful \\
for orientation fields) \\
Bifurcation, for the descrip- \\
tion of the collisions or splits \\
of different singular features \\
over space and time (useful \\
for orientation fields)
\end{tabular}

These descriptors depict different flow behaviors that can be observed in many applications. For instance, in texture synthesis and painterly rendering, the user often wants the texture patches and brush strokes to be oriented in a certain way. An orientation field can be created to achieve that with the desired instantaneous flow patterns prescribed by the specified streamlines. In crowd simulation, the user would like to steer a group of pedestrians to follow a certain route (or path). An advection field generated from the specified pathline can be applied to accomplish that (see Fig. 15). In a meteorological animation, the user may create the effect of two storm systems moving toward each other and eventually colliding (see Fig. 9). This can be done by controlling the movement (i.e., singularity paths) and interaction (i.e., bifurcation) of the two vortices in a time-varying vector field.

Note that for most graphics applications shown in this paper, instantaneous appearance is often more important than the exact path of a particle. For the rest of the paper, we will assume the designed fields serve as orientation fields except for the application of crowd simulation where the pathline design is used to generate an advection field. Nonetheless, for most examples the orientation fields are also used to advect the graphical primitives over time to achieve the effect of motion.

The overall pipeline of our system is as follows (Fig. 1). First, according to the selected design scenario, the user specifies a number of constraints. For key-frame design and field deformation, the focus is the creation of some instantaneous (static) fields. As such, specifying streamlines and singularities is sufficient. A streamline can be specified using the drawing tool of our system, which will compute the tangent vectors at the sample positions along the streamline as the constraints. For element-based design, pathlines, singularity paths, and bifurcations can be designed. In particular, for a pathline, besides computing the tangent vectors at the sampled positions, the temporal value for each sample point is required from the user (Section 5.1). The user is also responsible for providing the type for a singularity path (source, sink, or saddle) as a time-varying Jacobian. To specify a bifurcation, the user can describe a template function (Section 5.1) that will create the desired bifurcation. Note that in our system we only handle saddle-node bifurcation where a node is either a source or sink. Fig. 2 provides some examples on how the users can specify these flow descriptors with our system.

Once the constraints have been specified, our system generates a time-varying vector field by using the basis field summation (Section 5.2), constrained optimization (Sections 5.3 and 6.1), or time-varying transformation (Section 7) according to the selected design method. The resulting field is analyzed with singularities and bifurcations extracted. The user then has the ability to specify additional constraints or perform local topological editing in the form of singularity and bifurcation movement or cancellation. This process continues until the user is satisfied (Section 8).

In the next section, we will provide the mathematical definitions for the aforementioned flow characteristics.

\section{Time-varying Vector FieldS}

In this section, we briefly review the important concepts of time-varying vector fields, which will facilitate our later design tasks.

Streamlines and pathlines. We consider a 2-manifold $\mathbb{M}$. A time-varying vector field $V$ is a map $V: \mathbb{M} \times \mathbb{R} \rightarrow \mathbb{M}$, which can be expressed as a differential equation $\frac{d \mathbf{x}}{d t}=V(\mathbf{x} ; t)$. The solution of it given an initial state $\mathbf{p}_{0}=\left(\mathbf{x}_{0} ; t_{0}\right)$ is $\mathbf{x}(b)=$ $\mathbf{p}_{0}+\int_{0}^{b} V\left(\mathbf{x}(\eta) ; t_{0}+\eta\right) d \eta$, which is referred to as a pathline. It is the trajectory of the particle under $V$. The vector field $V\left(\mathbf{x} ; t_{c}\right)$ is an instantaneous vector field of $V$ at time $t_{c}$, which is steady. The solution from $\mathbf{p}_{c}=\left(\mathbf{x}_{\mathbf{c}} ; t_{c}\right)$ constrained in $V\left(\mathbf{x} ; t_{c}\right)$ is a streamline, and $\mathbf{x}(b)=\mathbf{p}_{c}+\int_{0}^{b} V\left(\mathbf{x}(\eta) ; t_{c}\right) d \eta$.

Instantaneous topology. The topology of $V\left(\mathbf{x} ; t_{c}\right)$ is referred to as the instantaneous topology of $V$ at $t_{c}$. It consists 


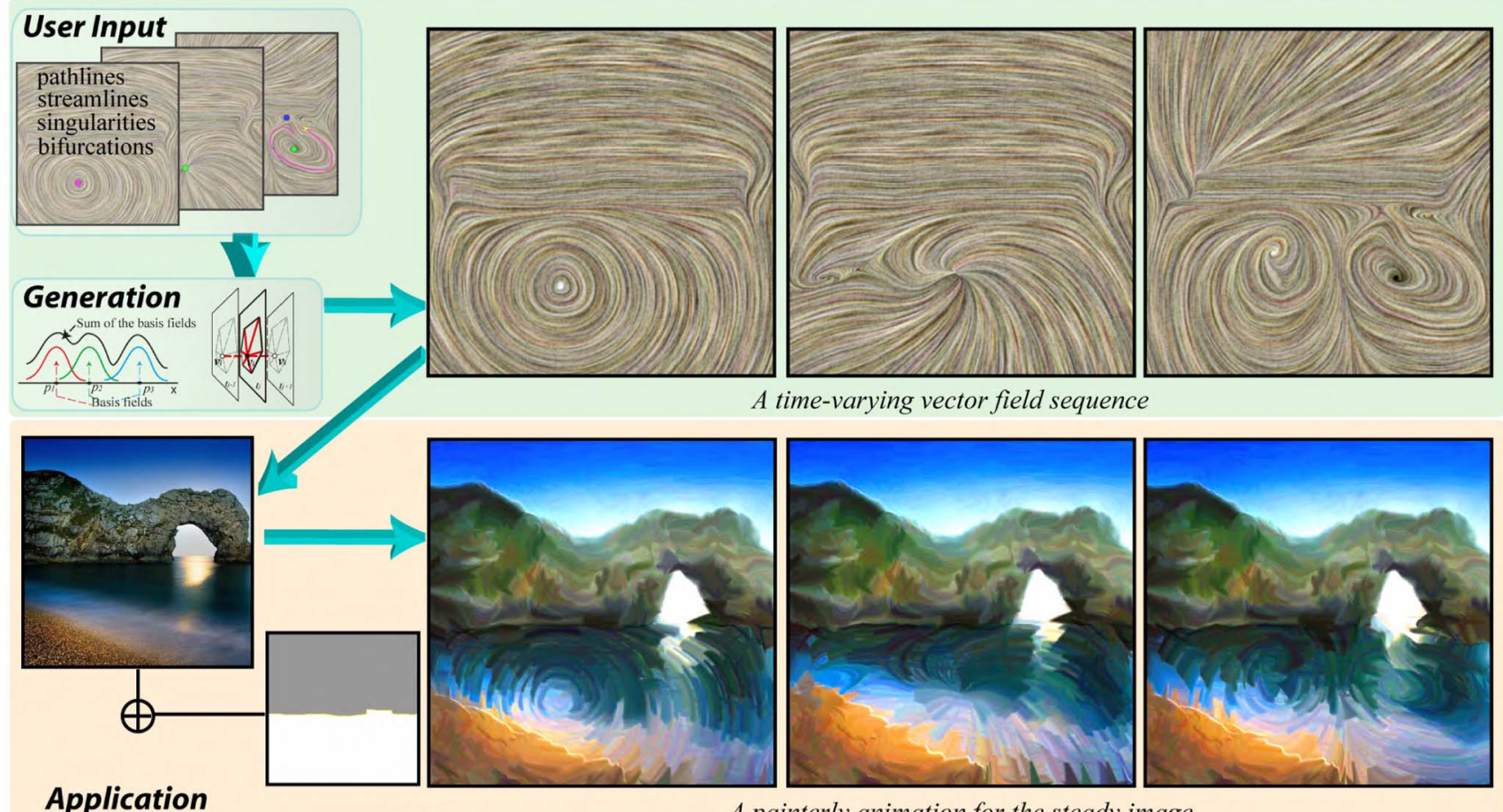

A painterly animation for the steady image

Fig. 1. This figure shows the pipeline of the presented design system for $2 \mathrm{D}$ time-varying vector fields. First, the user specifies the desired flow behaviors in the forms of spatial-temporal constraints. The system then produces a time-varying vector field that matches the constraints. The obtained field is then applied to computer graphics applications to create various dynamic effects. Here, we apply the obtained fields to produce painterly animation from a single image. Note that we use the created time-varying vector field to orient and move the brush strokes in the lower part of the image to achieve an artistic water wave effect: the vortex rotates, moves, and changes its characteristics, then splits into two vortices at the end. Please see the accompanying video for this animation, which can be found on the Computer Society Digital Library at http:// doi.ieeecomputersociety.org/10.1109/TVCG.2011.290.

of singularities, periodic orbits, and their connectivity [3] and describes the qualitative information of $V\left(\mathbf{x} ; t_{c}\right)$. This information has been applied to guide the creation and control of static vector fields [3], [8], [44], [51]. It has been shown that analyzing and tracking instantaneous features can provide more information for graphics applications than the space-time topology based on pathlines that is typically featureless [38]. Therefore, in the rest of the paper, we will make use of the notion of instantaneous topology to discuss the structural evolution of a time-varying vector field. Also, we focus on singularities only as they are relevant to the present graphics applications.

Singularities and singularity paths. A point $\mathbf{p}$ is called a singularity of $V\left(\mathbf{x} ; t_{c}\right)$ if $V\left(\mathbf{p} ; t_{c}\right)=0$. We are interested in the isolated singularities in the field, each of which can be enclosed by a compact neighborhood containing no other singularities. The type of each singularity is determined by the flow characteristics within this neighborhood. The linearization of $V\left(\mathbf{x} ; t_{c}\right)$ about $\mathbf{p}$ results in a $2 \times 2$ matrix $D V(\mathbf{p})=\left(\begin{array}{ll}\partial v_{x} / \partial x & \partial v_{x} / \partial y \\ \partial v_{y} / \partial x & \partial v_{y} / \partial y\end{array}\right)$ (called the Jacobian) which has two (potentially complex) eigenvalues $\sigma_{1}+i \mu_{1}$ and $\sigma_{2}+i \mu_{2}$. If $\sigma_{1} \neq 0 \neq \sigma_{2}$, then $\mathbf{p}$ is called a hyperbolic singularity. Observe that on a surface there are three types of hyperbolic singularities: sinks $\sigma_{1}, \sigma_{2}<0$, saddles $\sigma_{1}<0<\sigma_{2}$, and sources $0<\sigma_{1}, \sigma_{2}$. If $\sigma_{1}=\sigma_{2}=0, \mathbf{p}$ is a center. Any arbitrarily small perturbation will turn it to a hyperbolic singularity. Despite that, centers can still be structurally stable in a divergencefree field (e.g., an incompressible flow). Each singularity has a life span $\left[t_{s}, t_{e}\right]\left(t_{s}, t_{e} \in \mathbb{R}\right)$ where $t_{s}$ represents the time of its birth and $t_{e}$ is the time of its annihilation. The curve connecting each position of the singularity during its life span is called a singularity path. We assume the type of a singularity does not change during its life span.

Bifurcations. The birth and annihilation of singularities imply the change of the topological structure of the vector field. We refer to this qualitative change as the bifurcation and the places where these changes occur as the bifurcation points. Bifurcation is an important event in time-varying vector fields. In many graphics applications involving timevarying vector fields, bifurcations can lead to structural changes of certain geometry or properties, such as the splitting and merging of vortices in fluid animation. In some cases, these structural changes may cause visual artifacts. Fig. 3 shows an example where the break of texture structures induced by the bifurcations of the underlying vector field causes a visual discontinuity in the animation. Therefore, studying bifurcations and developing effective techniques to control them is necessary from the application perspective. The rigorous definition of bifurcation is beyond the scope of this paper. However, a necessary condition for a bifurcation to occur is that at a bifurcation point $\mathbf{p}_{\mathrm{b}}$, the Jacobian of the vector field $D V\left(\mathbf{p}_{\mathbf{b}}\right)$ is singular, i.e., its determinant is zero. In the meantime, $\left\|V\left(\mathbf{p}_{\mathbf{b}}\right)\right\|=0,\left\|\frac{\partial V\left(\mathbf{p}_{\mathbf{b}}\right)}{\partial t}\right\| \neq 0$, and $D^{2} V\left(\mathbf{p}_{\mathbf{b}}\right)$ is nonsingular. The corresponding proofs and more comprehensive introduction of the bifurcation theory can be found in [11] Consistent with our focus on singularities, in this paper we discuss only local bifurcations, such as saddle-node (fold) 


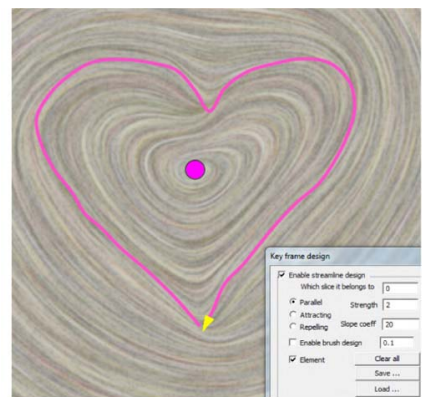

(a)

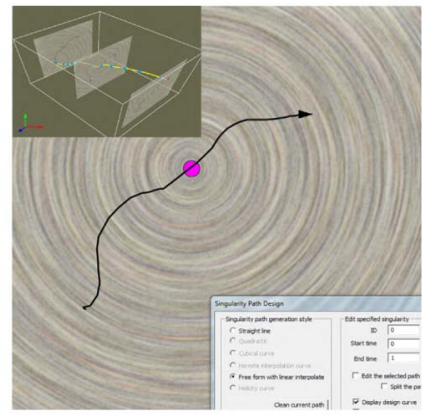

(c)

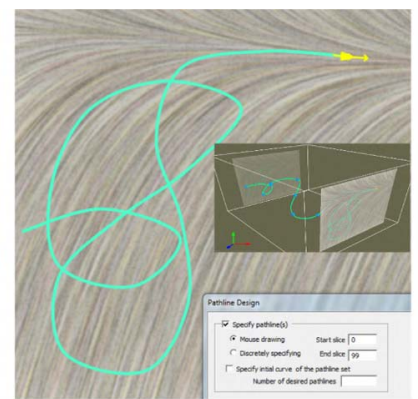

(b)

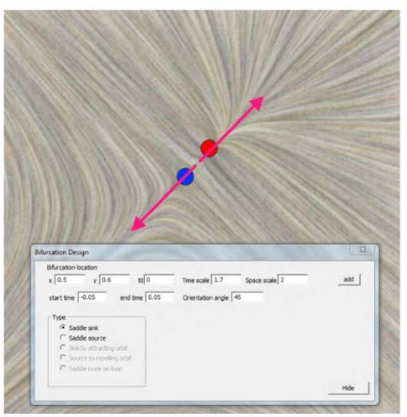

(d)
Fig. 2. Our user interface showing different design metaphors: (a) Streamline. (b) Pathline. (c) Singularity path. (d) Bifurcation. A streamline is specified at a particular time as a 2D curve. A pathline can be provided either in the $2 \mathrm{D}$ domain with the starting and ending time information or directly in the spatial-temporal domain (see the inset of $b$ ). Similarly, a singularity path can be designed in either 2D domain with the birth and death times or in the spatial-temporal domain (see the inset of c). A bifurcation is prescribed as a point in the spatial-temporal domain with the coordinate, scaling, and orientation information.

bifurcation and its inverse bifurcation which refers to the annihilation of sink/source and saddle pairs. Fig. 4 illustrates a saddle-source bifurcation where a source with Poincare index 1 and a saddle with index -1 move toward and finally cancel each other over time. This bifurcation can be formulated as follows [11]:

$$
V((x, y) ; t)=\left(\begin{array}{c}
t+x^{2} \\
y
\end{array}\right)
$$

while a saddle and sink creation bifurcation can be formulated as follows:

$$
V((x, y) ; t)=\left(\begin{array}{c}
t-x^{2} \\
-y
\end{array}\right) .
$$

The change of the type of a singularity corresponds to a transcritical bifurcation, for instance, sink $\rightarrow$ center $\rightarrow$ source, and vice versa. When this occurs, we consider a new singularity is born while the old one is eliminated.

With these concepts, we next describe how we support the three different design scenarios as introduced in Section 1. We first describe the setting of our computation domain.

Computation domain. We consider a subdomain $\mathbf{D}_{\mathbf{X}}=$ $(X ; t)$ where $\mathbf{D}_{\mathbf{X}} \subset \mathbb{M} \times \mathbb{R}$ is a spatial-temporal domain. $X$ is a triangulation of a $2 \mathrm{D}$ curved surface embedded in 3D, and $t \in[0,1]$ is the time parameter. To represent and store the field, we discretize $t$ evenly. We denote these discretely sampled values as $\left\{t_{j}\right\}$. We then compute and store the instantaneous fields at these discrete times $\left\{t_{j}\right\}$ in order. In each instantaneous field, vector values are sampled at the

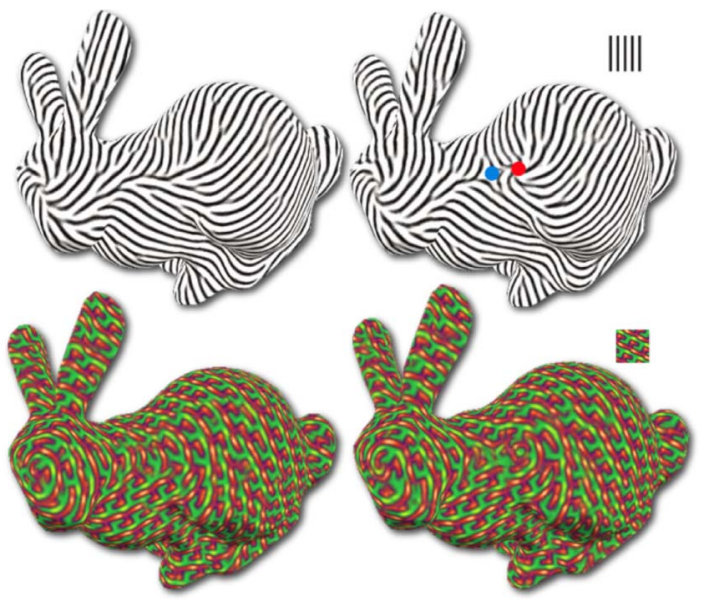

Fig. 3. This figure shows an example of saddle-node bifurcation in an orientation vector field. The creation of a pair of saddle and sink causes the break of texture structure on the back of the bunny. Note that we sample the two frames before (left column) and after (right) the bifurcation point to reveal the discontinuity.

vertices of the triangulation $X$. The inset figure shows such a configuration. For a planar domain, we use a free-form boundary. Along $t$, we assume the time-varying vector field in $\mathbf{D}_{\mathbf{X}}$ is a portion of the time-varying field with $t \in(-\infty, \infty)$. Other boundary conditions of $t$ can be employed, such as the periodic boundary conditions often used in fluid simulation [35].

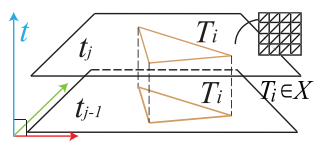

In order to enable a more flexible speed control of the final animation sequences, the parameter $t$ has a linear relation with the physical time, that is, $c \cdot \mathrm{d} t\left(c \in \mathbb{R}^{+}\right)$will be the actual time interval when applying the created field to the target applications.

Interpolation scheme. We assume that the vector field is defined on the vertices of the mesh domain. In space $X$, the vector values within a triangle are computed using the parallel transport technique of Zhang et al. [51]. Along the parameter $t$ dimension, we employ a similar interpolation technique proposed by Tricoche et al. [40] to guarantee the linearity over $t$. In particular, the vector value of a sample point $p$ in-between two succeeding frames can be computed using linear interpolation of the two values at $p$ at the two frames.

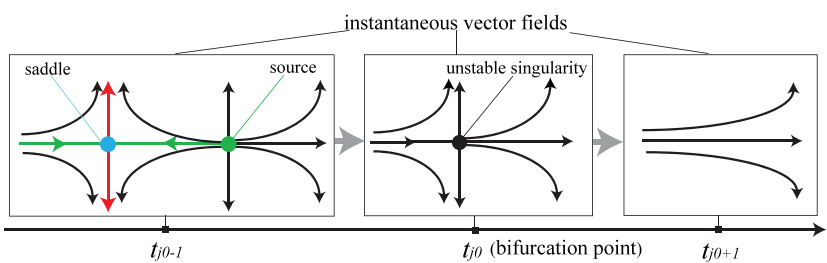

Fig. 4. This example demonstrates a saddle-source cancellation bifurcation. The directional curves illustrate the flow behavior. Two singularities are shown in the left at $t_{j_{0}-1}$. They move toward each other when $t$ increases and collide at $t_{j_{0}}$ (middle). The two singularities are canceled after they meet, which results in a singularity-free vector field at $t_{j_{0}+1}$ (right). 


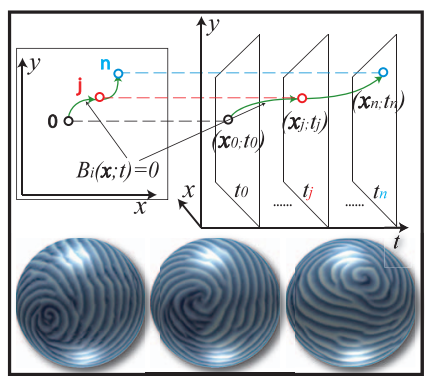

Fig. 5. Singularity path.

\section{Element-Based Design}

In this section, we describe how we support the design of local spatial and temporal behaviors of the flow through a number of design elements that can be extracted from the user-specified flow characteristics. These design elements are later combined to generate a time-varying vector field.

\subsection{Design Elements}

Our system supports the following design elements.

Singular elements. Modeling singular elements is an essential functionality for vector field design. We extend the singular elements in the static field design [51] to our spatial-temporal setting. Specifically, we denote a singular element as $S(J, P(t), M(t))$, where $J$ is the Jacobian that determines the type of the singular element, $P(t)$ represents the path of the singular element over time, and $M(t)$ is the affine transformation matrix (i.e., scaling and rotating) that is exerted on the element along $P(t)$. We assume $J$ is fixed along $P(t) . P(t)$ is derived from a user-specified path (Fig. 5). In particular, after the user sketches the path of a singular element, a Hermite spline curve is fitted to it to form a smooth path $P(t) . M(t)$ is initialized as an identity matrix and can vary along $P(t)$. Given a time $t_{c}, M\left(t_{c}\right)=$ $\left(\begin{array}{cc}s_{x}\left(t_{c}\right) & 0 \\ 0 & s_{y}\left(t_{c}\right)\end{array}\right) R\left(\theta\left(t_{c}\right)\right)$ where $s_{x}\left(t_{c}\right)$ is an $x$ scaling, $s_{y}\left(t_{c}\right)$ a $y$ scaling, and $R\left(\theta\left(t_{c}\right)\right)$ a rotation centered at $P\left(t_{c}\right)$. The user specifies a number of $M\left(t_{i}\right)$ at the desired times $t_{i} . M(t)$ can then be computed through linearly interpolating $s_{x}\left(t_{i}\right)$ and $s_{x}\left(t_{i+1}\right), s_{y}\left(t_{i}\right)$ and $s_{y}\left(t_{i+1}\right)$, and $\theta\left(t_{i}\right)$ and $\theta\left(t_{i+1}\right)$ where $t_{i}<t<t_{i+1}$.

$P(t)$ starts and ends at $t=0$ and 1 by default. If it starts or ends in between, a certain bifurcation is induced, which in turn involves another singularity with an opposite Poincaré index. In design, this can be achieved by intersecting the two singularity paths (by definition in Section 4). At the bifurcation point where the two paths intersect, the local Jacobian is singular with eigenvalues of zero (Section 4), while the Jacobian of the rest of the field is not. That said, if the singular Jacobian is used to generate a global field as we create a field with a singularity [51], the obtained field will not be continuous. In addition, the Jacobian at the point $\mathbf{p}_{\mathbf{b}}$ where the bifurcation occurs is not singular before and after bifurcation. Therefore, using the varying Jacobian at $\mathbf{p}_{\mathbf{b}}$ to generate a sequence of static fields will result in large variance in the obtained fields, i.e., discontinuity of the flow patterns can be observed before, at, and after bifurcation. To address this issue, we introduce the bifurcation elements that describe the globally smooth

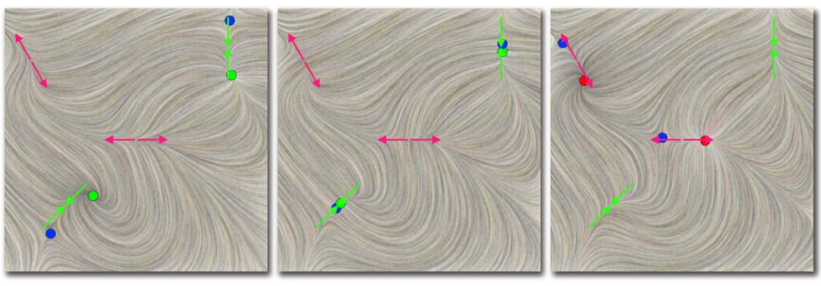

Fig. 6. A number of bifurcations are inserted using (2) (red) and (1) (green). The arrows show the bifurcation directions.

flow behavior over time under the presence of the corresponding bifurcations.

Bifurcation elements. Recall that we are concerned with saddle-node bifurcations in this paper. Equations (1) and (2) are two normal forms that define a saddle-node bifurcation at position $(0,0 ; 0)$ in domain $X$ (i.e., a bifurcation element). Specifically, (1) induces a saddle-source cancellation and (2) defines a saddle-sink creation. During design, the user prescribes the position, $\left(x_{0}, y_{0} ; t_{0}\right)$, of a bifurcation with the desired type in domain $X$. Thus, we replace $x=x-x_{0}$, $y=y-y_{0}$, and $t=t-t_{0}$ in (1) or (2) to place the bifurcation elements in the right position. Further, a user-controlled transformation can be exerted to scale the range of the bifurcation in both space and time and reorient an axis (a straight line in this case) to control where and how the bifurcation occurs along the axis. Fig. 6 provides an example where the user inserts a number of bifurcations.

These bifurcation elements enable the user to insert bifurcations through certain templates (i.e., the bifurcation normal forms) with guaranteed smooth transition in space and time. However, it does not allow modification of the paths of the involving singularities. A more intuitive and flexible design interface for bifurcations is much desired and should be studied in the future work.

Regular elements. In static field design, a regular element is useful in providing the translation or advection direction for a particle located at a point and is related to streamlines. In the design of time-varying vector fields, this element is tightly linked to pathlines.

We define a regular element as $R(V(t), P(t))$ where $P(t)$ is a prescribed pathline and $V(t)$ is the tangent direction at $P(t)$ in space and at a time $t$.

Consider a user-specified pathline curve which consists of the positions of a particle $\mathbf{p}$ from $t_{s}$ to $t_{e}\left(t_{e} \geq t_{s}\right)$, denoted by $\bigcup_{t_{s}}^{t_{e}}(\mathbf{p}(s))$. Assume $m$ sample points, $\mathbf{p}_{i}$, along the curve are taken. A Catmull-Rom spline $P(t)$ is computed with $\left\{\mathbf{p}_{i}\right\}$ as the control points. The spline curve is densely sampled as the set of evenly spaced points $\left\{\mathbf{s p}_{j}\right\}$. Assume $K$ is the number of sample points on the spline curve and $N$ is the number of time samples. We set $K>4 N$ for a smooth representation such that $V\left(t_{i}\right)=\left(\mathbf{s p}_{j}-\mathbf{s p}_{j-1}\right)$, a good approximation of the tangent direction, is placed at $P\left(t_{i}\right)$ where $t_{i} \in[0,1]$ is the $i$ th sampled time (see Fig. 7). To reduce user input, a uniform sampling, $t_{i}=t_{s}+i \times\left(t_{e}-\right.$ $\left.t_{s}\right) /(N-1)$ can be used. However, this is not required. $\mathbf{s p}_{j}$ and $\mathbf{s p}_{j-1}$ are the points that enclose $P\left(t_{i}\right)$ on $P(t)$.

\subsection{Basis Fields Summation}

In order to generate a time-varying vector field from the user-specified elements described above, a basis field 


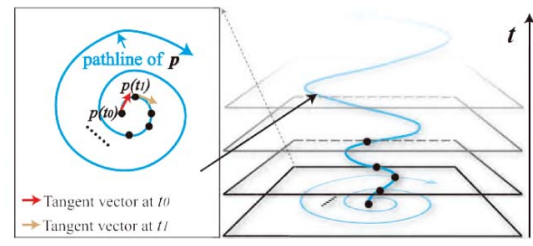

Fig. 7. Pathline example.

summation can be used which has been applied to static field design [47], [44], [51]. We extend this basis field summation to take into account the design elements with time-varying characteristics introduced in the previous section. Specifically, the basis field generated by a singular element at time $t$ has the form:

$$
V_{i}(\mathbf{x} ; t)=e^{-d\left\|\mathbf{x}-\mathbf{p}_{i}(t)\right\|^{2}} M_{i}^{T}(t) J_{i} M_{i}(t)\left(\begin{array}{c}
x-x_{\mathbf{p}_{i}}(t) \\
y-y_{\mathbf{p}_{i}}(t)
\end{array}\right),
$$

where $\mathbf{p}_{i}(t)=\left(x_{\mathbf{p}_{i}}(t), y_{\mathbf{p}_{i}}(t)\right)$ is the position of the singular element at time $t$ along the path $P_{i}(t)$, and $M_{i}(t)$ is the transformation acting on $J_{i}$. The basis field for a regular element given time $t$ has the form

$$
V_{i}(\mathbf{x} ; t)=e^{-d\left\|\mathbf{x}-\mathbf{p}_{i}(t)\right\|^{2}} V_{i}(t) .
$$

A bifurcation element generates the following basis field:

$$
V_{i}(\mathbf{x} ; t)=e^{-d\left\|\mathbf{x}-\mathbf{p}_{i}(t)\right\|^{2}} V_{b i}\left(M_{i}\left(\begin{array}{c}
x-x_{\mathbf{p}_{i}}(t) \\
y-y_{\mathbf{p}_{i}}(t)
\end{array}\right) ; t-t_{i}\right),
$$

where $\left(\mathbf{p}_{i}\left(t_{i}\right) ; t_{i}\right)$ is the position at which the $i$ th bifurcation occurs and $M_{i}$ is a transformation matrix specified by the user to orient the moving direction of the two singularities. $V_{b i}$ is one of the bifurcation normal forms (e.g., (1) and (2)).

Accordingly, the obtained global time-varying vector field is the sum of these individual basis fields

$$
V(\mathbf{x} ; t)=\sum_{i} V_{i}(\mathbf{x} ; t) .
$$

Fig. 8 provides a time-varying vector field generated using the element-based design and the basis field summation. Note that we extended the design elements to the space-time domain from their static counterparts. Each design element at a given time acts as a static one except for a bifurcation element that is defined by its normal form over time. To that end, the basis field summation is largely the same as its static counterpart. Consequently, the issue of the cancellation of an element by the influence of its nearby elements can arise. To relieve that, we can use a sharper falloff function with a larger $d$ value or require the design elements to be placed sufficiently far apart to reduce their mutual influence. Another possible solution is to extend the work of Turk and O'Brien [42] for surface modeling (i.e., scalar function modeling) to basis field summation. One could determine the weight for each basis field at a vertex and compute a weighted sum of the basis fields instead of a uniform sum. It is hoped this would preserve the prescribed features. However, it is unclear whether such an extension is easy to devise and how well it will work for vector data. In this work, we resort to constrained optimization, a popular vector field generation technique for static field design [6], [8], [34], [49]. In particular, the constraints are set at the

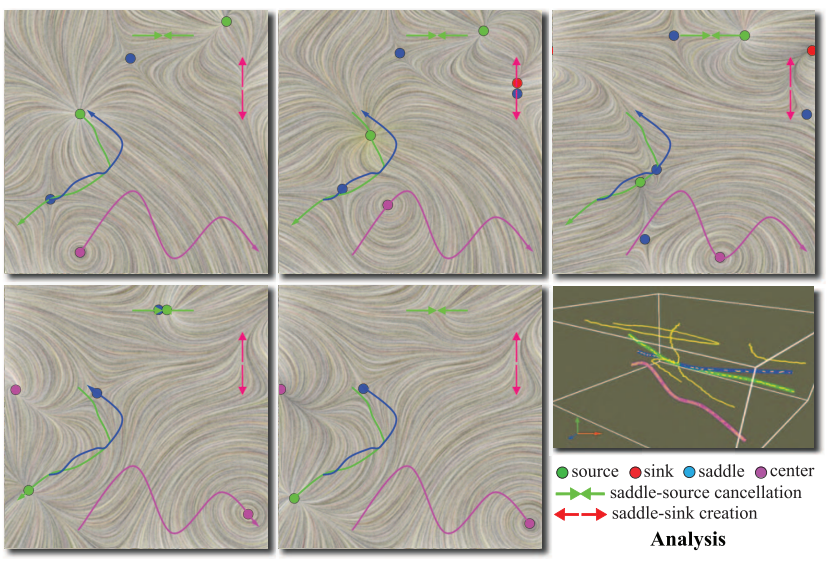

Fig. 8. A time-varying vector field generated using a number of design elements. The instantaneous fields are ordered from left to right and top to the bottom. The singularity paths of the singular elements are highlighted as the colored curves (green for source, blue for saddle, and magenta for center). Two saddle-node bifurcations are also inserted. The obtained field has smooth change over time as shown in the plot of the lower right. In addition to the desired singularities and bifurcations, there are also unexpected singularities and bifurcations as shown in the analysis, due to the nature of the basis field summation approach.

boundaries of a number of small and compact regions that enclose the design elements (see the inset). Note that the regions that contain the prescribed elements need to be isolating (i.e., not overlapping with each other) in order to preserve the desired features, due to our discrete setting and elected linear interpolation scheme.

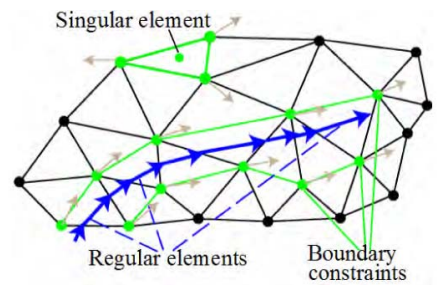

\subsection{Constrained Optimization}

In static field design, constrained optimization solves for a harmonic vector field which satisfies the Laplacian system $\Delta \overrightarrow{\mathbf{V}}=\overrightarrow{0}$ where $\Delta=\nabla^{2}$ is the discrete Laplace operator and $\overrightarrow{\mathbf{V}}$ is the unsolved vector field [51]. Specifically, given a region $N$ of a triangular mesh where the vector values at the boundary vertices of $N$ are the constraints, the constrained optimization has the form of

$$
\bar{V}\left(v_{i}\right)=\sum_{j \in J} \omega_{i j} \bar{V}\left(v_{j}\right)
$$

where $v_{i}$ is an interior vertex, $v_{j}$ 's are its adjacent vertices in $N \cdot \bar{V}(v)$ represents the average vector value at vertex $v \cdot \omega_{i j}$ is the weight between vertex $v_{i}$ and $v_{j}$. Note that we consider the boundary condition of Dirichlet type. Equation (7) is a sparse linear system in the form of $A \overrightarrow{\mathbf{x}}=\overrightarrow{\mathbf{b}}$. For fast solution, one can use a uniform weighting scheme or mean curvature weighting [49] which guarantees $A$ to be a symmetric positive definite (s.p.d.) matrix such that the state-of-the-art Cholesky factorization solver can be applied to solve it efficiently [48], [49]. In this case, we assume the vector values at vertices are expressed under the $3 \mathrm{D}$ global coordinate system. The solution of this setting will result 
in vector fields that do not always reside in the tangent space for a curved surface. Although we can project these vector fields to their tangent space, the projected fields usually contain many unexpected singularities. In order to produce a tangential vector field with better quality (i.e., fewer unexpected singularities), we recommend the technique of parallel transport used in [27] to construct the Laplacian system in tangent space directly

$$
\bar{V}\left(v_{i}\right)=\sum_{j \in J} \omega_{i j} T_{i j} \bar{V}\left(v_{j}\right),
$$

where $T_{i j}$ is the transformation matrix for parallel transport along an edge $\left(v_{i}, v_{j}\right)$. This will give rise to a non-s.p.d. matrix. To solve it, we use a biconjugate gradient approach [32]. This also provides the foundation for our later extension to solve for the spatial-temporal problem.

Given the constrained optimization, the time-varying vector field can be generated by solving a sequence of Laplacian systems with the boundary constraints set according to the instantaneous characteristics of the prescribed elements at the sampled times.

Although the constrained optimization can better preserve the specified features at single time steps as long as an isolating region can be found for each feature, it is still challenging to preserve them over time. This is because the features are moving and changing over time, and if two features are getting too close to each other, it is difficult to compute the isolating regions that enclose them. Another limitation for the constrained optimization is, when bifurcations occur, simply specifying the vector values at the boundaries of the neighborhoods that contain the bifurcation points is not sufficient due to the degeneracy previously mentioned. Because of this issue, the basis field summation is still used in the present system for bifurcation design.

In the next two sections, we will describe two different design scenarios that complement the element-based design.

\section{Key-Frame Design}

Given our discrete setting of time-varying vector fields, it is natural to prescribe the flow behavior in certain time steps and ask the system to create a time-varying vector field that smoothly transitions from one state to the next. This leads to the key-frame design. This design scenario is useful when a number of critical time steps need to be designed to achieve the desired behaviors while the others are not so important. It is a widely used technique in the computer animation community to efficiently generate animation sequences.

The key-frame vector fields can be designed using any existing static vector field design techniques [6], [8], [34], [51]. In order to generate the rest of the time-varying vector field from the given key-frame fields, some vector or anglebased interpolation can be employed. However, using interpolation can create degenerate instantaneous fields (using vector-based linear interpolation) or discontinuities due to angle ambiguity (using angle-based interpolation). To address that, we introduce a spatial-temporal constrained optimization, an extension of the approach in Section 5.3.

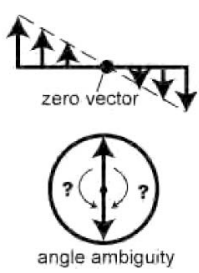

\subsection{Spatial-Temporal Constrained Optimization}

Similar to the static case, we define an extended spatialtemporal Laplacian system by taking into account the additional parameter $t$. Note that discrete Laplacian system is constructed by considering the relation between spatially connected vertices. This can be extended to higher dimensions. Based on this observation, we treat $t$ as the additional dimension in space and assume a direct connection between two neighboring vertices that are the two copies of the same vertex of triangulation $X$ at two succeeding times. The image below shows such a configuration. Given a vertex $\left(\mathbf{v}_{i} ; t_{j}\right)$ of $X$, consider a stencil shown in the figure. We assume there are (virtual) edges connecting $\left(\mathbf{v}_{i} ; t_{j}\right)$ and $\left(\mathbf{v}_{i} ; t_{j-1}\right),\left(\mathbf{v}_{i} ; t_{j}\right)$, and $\left(\mathbf{v}_{i} ; t_{j+1}\right)$, respectively. We solve a spatial-temporal Laplacian $\nabla \sum_{j} \omega_{j} V=\overrightarrow{\mathbf{0}}$ where $\sum_{j} \omega_{j} V$ represents the weighting sum of the time-varying vector field over $t$. This is equivalent to solving the following linear system:

$$
\begin{aligned}
\omega \bar{V}\left(\mathbf{v}_{i} ; t_{j}\right)= & \sum_{k \in N(i)} \omega_{i k} T_{i k} \bar{V}\left(\mathbf{v}_{k} ; t_{j}\right)+\omega_{j, j-1} \bar{V}\left(\mathbf{v}_{i} ; t_{j-1}\right) \\
& +\omega_{j, j+1} \bar{V}\left(\mathbf{v}_{i} ; t_{j+1}\right),
\end{aligned}
$$

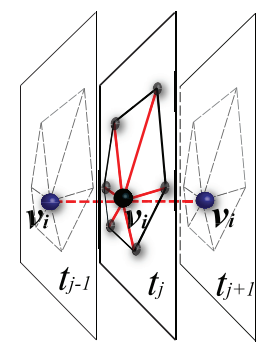

where $N(i)$ denotes the one-ring neighbors of $\left(\mathbf{v}_{i} ; t_{j}\right)$, $\bar{V}\left(\mathbf{v}_{i} ; t_{j}\right)$ represents the average vector value at position $\left(\mathbf{v}_{i} ; t_{j}\right) . \omega_{j, j-1}$ and $\omega_{j, j+1}$ are positive weights determining how fast the relaxation is along $t$. In our implementation $\omega_{j, j-1}=\omega_{j, j+1}=b \sum_{k} \omega_{i k} \cdot \omega=\sum_{k \in N(i)} \omega_{i k}+\omega_{j, j-1}+\omega_{j, j-1}$ is the normalization coefficient. $b$ controls the speed of relaxation along $t$. We use $b=30$ for all examples. We point out that this formula can be further extended by considering more sampled steps along the $t$ axis to achieve smoother results as bi-Laplace smoothing does in static case [8]. For better quality, we use parallel transport to solve for tangential vector fields with mean value weights [9].

With the spatial-temporal constrained optimization, a bifurcation can be implicitly created by specifying the instantaneous fields before and after bifurcations, e.g., the left and right fields in Fig. 4. However, the user is not able to specify the exact paths for both singularities, which can be achieved by the element-based design. Fig. 9 shows a timevarying vector field generated using key-frame design. It demonstrates that two vortices move toward each other, finally collide, and merge into one. 

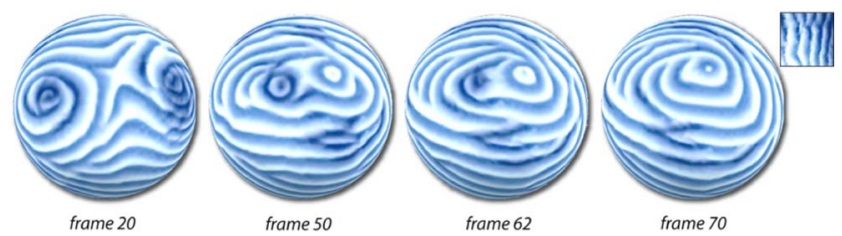

Fig. 9. This image shows a number of frames from a texture animation on sphere which simulates the collision of two storm systems. The animation is driven by an orientation field and an advection field. Both are designed using the techniques introduced in this paper. Frames 1 (not shown), 50, and 100 (not shown) are the key frames.

\section{Time-Varying Field Deformation}

In some design cases, the user is only given or has to start with an existing static field to generate a sequence of continuously changing fields over time. One way to achieve that is to gradually deform the initial field. This deformation process can be performed through physically based simulation as one uses in fluid simulation. To incorporate the initial field in the simulator, it has to be considered as some external force field to start the simulation. The initial field has only indirect influence to the obtained sequence. This is not always desirable if the user prefers a time-varying vector field, that is, neither incompressible nor physically plausible. Therefore, other more intuitive and flexible approaches need to be explored. In this section, we propose a simple way to deform the initial field by using a global time-varying transformation, i.e., a matrix whose entries are functions of time. We refer to this approach as the matrix-based design.

Our system assists such design by exerting a timedependent transformation matrix on the initial field, $V(t)=M(t) V\left(t_{0}\right) . M(t)$ is an affine transformation of the form $\left(\begin{array}{ll}M_{11}(t) & M_{12}(t) \\ M_{21}(t) & M_{22}(t)\end{array}\right)$, where $M_{i j}(t)$ are some functions of $t . M(t)$ can be designed through the graphics interface by specifying the $x$ scaling, $y$ scaling, and rotation similar to what has been described in Section 5.1. Our system also provides a text editor interface to allow the user to directly provide the numeric values for the four entries. The transformation matrix at $t_{i}$ is then computed through linearly interpolating the identity matrix and the user-specified one. However, such random specification of transformation can easily lead to degenerate (e.g., zero or discontinuous) fields. Matrixbased design has its own value where the transcritical bifurcations can be achieved easily by rotating the Jacobian of the singularities over time. For instance, a transcritical bifurcation (i.e., source $\rightarrow$ center $\rightarrow$ sink) is induced at the belly of the Buddha (Fig. 10).

In addition to transforming the whole field, our system also allows the user to select one or more representative streamlines computed from the initial field and continuously transform them over time. The deformed representative streamlines are then used to generate new instantaneous fields at each sampled time using the static field generation techniques mentioned earlier. The inset figure shows an example of a representative streamline (shown in magenta) deformed over time. A surface connecting the new position of this streamline with the preceding one is shown. The intersection of this surface with the $t=t_{i}$ plane is a smooth curve representing the

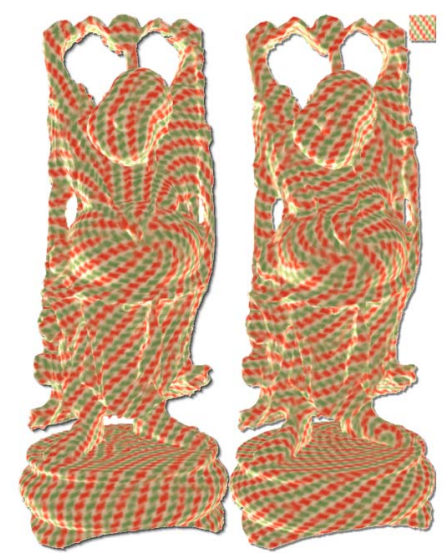

Fig. 10. A transcritical bifurcation at the belly of the Buddha using field deformation.

streamline at $t_{i}$, which is used to generate an instantaneous field. However, more elegant and sophisticated techniques should be devised to refine the deformation process and achieve more flexible and controllable results, which we will leave for the future work.

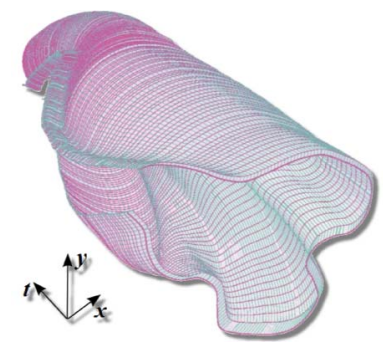

\section{Local Topological Editing}

Editing functionality is required for a design system because of the appearance of undesired features such as singularities and bifurcations in the generation phase. Our system provides the user with a number of options to edit a given time-varying vector field. First, the user can edit the instantaneous fields to modify the time-varying vector field at specific times. Second, the bifurcations can be canceled or moved under certain conditions.

Instantaneous field topological editing. Given the instantaneous field at a particular time $t$, the user can remove two unwanted singularities using the simplification techniques of Chen et al. [3]. This instantaneous field is then considered as a key frame for the regeneration of the field.

\subsection{Bifurcation Editing}

We have demonstrated the relations between saddle-node bifurcations and the structural changes in texture animations. We now describe techniques to control them. To do so, the system first extracts bifurcations from the designed fields using the techniques proposed by Tricoche et al. [40], and then provides two editing operations for the user.

Bifurcation removal. Certain bifurcations can be removed. First, the user can remove the isolated bifurcations. According to our setting (Section 4) and the Poincare theorem, these isolated bifurcations can only involve singularities that start or end at the boundary of our 


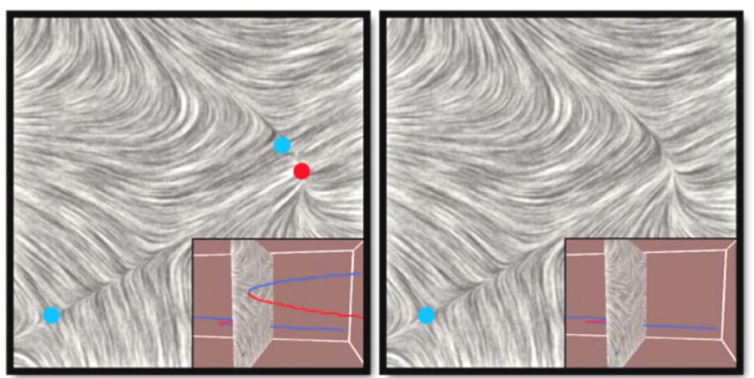

Fig. 11. Example of bifurcation editing.

computation domain $\mathbf{D}_{\mathbf{X}}$. Other isolated bifurcations involve singularities whose paths form loops. The top row of the inset shows the removable isolated bifurcations. To remove them, we simply cancel the pairs of the involving singularities [3]. Second, consider three singularities $p_{i}$ $(i=1,2,3)$ with intervals of existence $(0, \beta),(\alpha, \beta),(\alpha, 1)$, respectively. Assume a saddle-node bifurcation between $p_{1}$, $p_{2}$ at $\beta$, and a saddle node bifurcation between $p_{2}$ and $p_{3}$ at $\alpha$. We then can remove both bifurcations and retain only one singularity. The bottom row of the inset shows such an example. The two bifurcations that are connected by the blue curve (the path of a saddle) can be collapsed. Fig. 11 shows an example of saddle-node bifurcation removal. More complex control of nonisolated (i.e., connected) bifurcations is possible, which is beyond the scope of this paper.

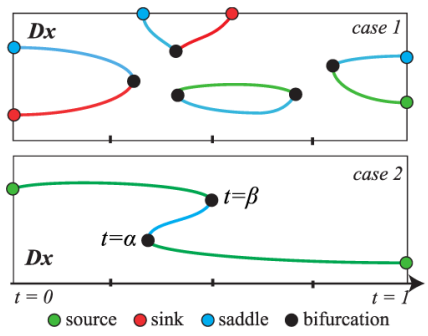

Bifurcation movement. Similar to singularities, a bifurcation can be moved using our system. This can be achieved by moving the involving singularities over space at particular time $t$. The edited instantaneous field is then set as a key frame. The spatial-temporal constrained optimization will smooth the rest of the field. Note that the movement of these two singularities should obey the topological constraints proposed by Zhang et al. [51]. This guarantees that no other topological features are affected during the movement. This functionality is particularly useful when the bifurcation is not isolated and causes visual discontinuity (e.g., Fig. 3). The bifurcation movement could move it to a nonvisible part of the object.

General global smoothing over the spatial-temporal domain is also available, similar to the smoothing scheme of [18] for fining the edge fields, i.e., some tensor fields, in the application of painterly rendering of videos.

\section{Applications and Discussion}

In this section, we present a number of graphics applications that can benefit from the time-varying vector fields generated using the proposed techniques.
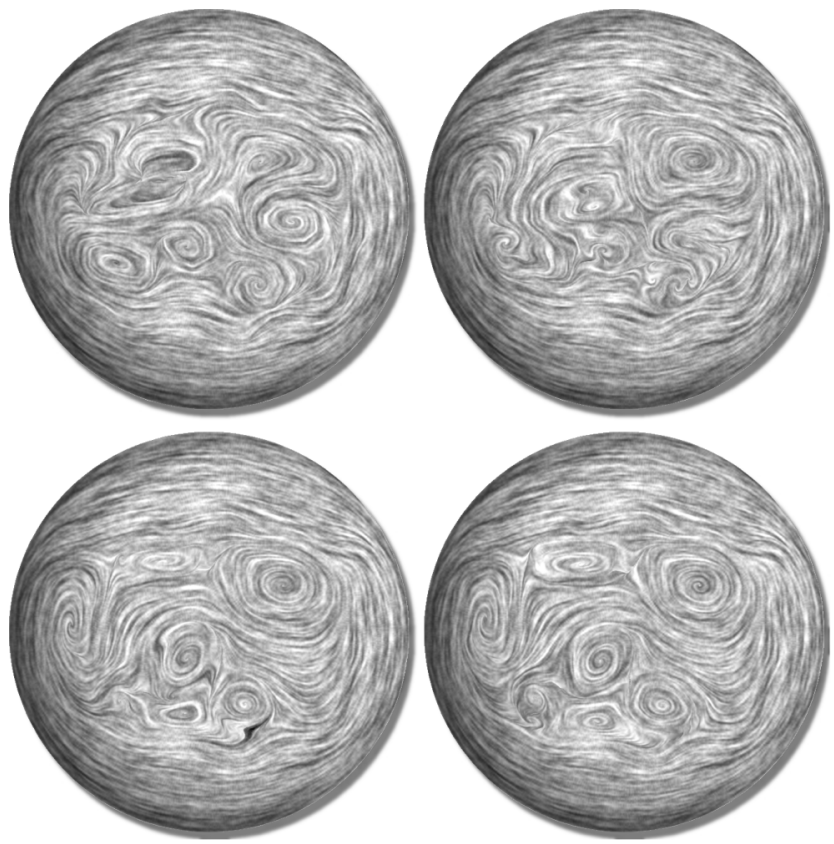

Fig. 12. A time-varying incompressible flow on the sphere.

\subsection{Texture Synthesis and Animation}

We have applied the designed time-varying fields to create a number of synthetic texture animations (Figs. 3 and 9). Flowguided texture synthesis and advection has been introduced to the visualization community for dense flow visualization by van Wijk [44], [43], Laramee et al. [22], and Neyret [26]. Kwatra et al. [20] present an optimization-based plane texture synthesis which can be used for flow-guided texture animation. Lefebvre and Hoppe [23] introduce an appearance-space texture synthesis technique that can handle texture advection over static surfaces. Han et al. [13] extend the work of [20] to 3D mesh surfaces. Later, Kwatra et al. [19] and Bargteil et al. [2] extend the texture advection techniques to the problem of fluid texturing on surfaces. Recently, Ma et al. [24] introduce a texture synthesis technique for flow patterns to create a more detailed synthetic texture and animation. In this paper, we employ the technique of Kwatra et al. [19]. To apply the created timevarying vector fields, we make use of the instantaneous snapshots of the time-varying vector fields to orient and move the texture patches on surfaces.

\subsection{Creation of Dynamic Scenes}

Our system can be used to create fluid effects on surfaces through proper design and setting of the boundary conditions. Fig. 12 shows an incompressible flow on the sphere generated using our system. In addition, the present system allows the creation of more complex dynamic effects such as wind writing on a meadow (Fig. 13), and the advection of leaves in the fluid flow with self-spinning effect (Fig. 14). In Fig. 13, instead of adding physically realistic winds, we design a time-varying vector field that mimics writing on the grass. To achieve that, the user first specifies the flow to represent the writing of the letters. The system automatically records the vector fields as key frames during the sketching of these letters. The spatial-temporal constrained optimization is then used to solve for a time-varying vector field. Each 

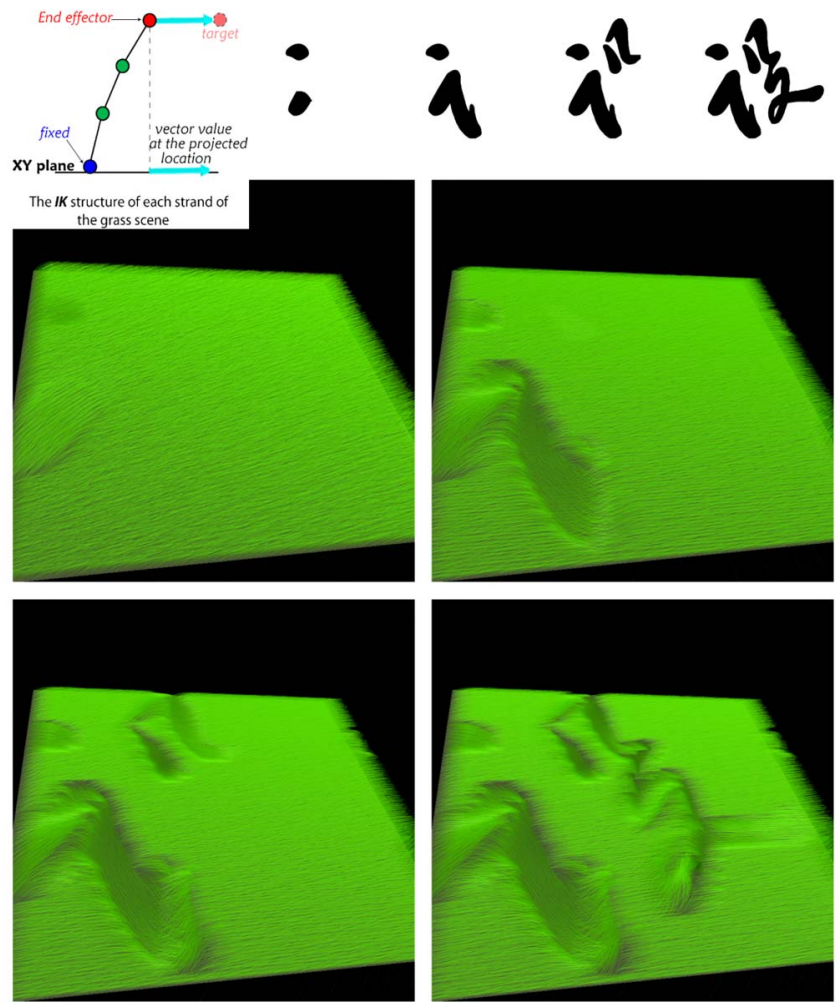

Fig. 13. An animation of writing on grass. The dynamics of the grass is driven by a created time-varying vector field. The grass consists of over 32,000 strands, each of which has the structure shown in the top left corner.

strand of the grass is represented as a rigid body skeleton. The bottom of the skeleton is fixed on the ground while the top node is manipulated by a force field which is the created timevarying vector field. The movement of this skeleton is computed by an inverse kinematic solver [28]. The grass is rendered using the technique of illuminated lines [25]. The field used to drive the movement of the leaves (Fig. 14) was created through element-based design. The spinning effect is achieved by maintaining a constant angle between the up direction of a particle and the advection direction at the given position.

\subsection{Steerable 2D Crowd Animation}

Crowd simulation is an important technique in games, movies, and urban planning. There are two groups of crowd simulation techniques: agent-based and force-based. While agent-based methods can provide more detailed and realistic simulation, it is still prohibitively expensive to simulate a large number of pedestrians with a complex environment. In contrast, the force-based technique considers the pedestrians in the crowd as particles. Their movement is determined by computing the gradient of a potential field by taking into account the environment and neighboring people. This method is fast at the expense of losing the detailed behavior of the individual pedestrians. Both approaches support the control of initial states yet lack of the continuous steering of the crowds over time. Recently, Patil et al. [29] propose the use of a navigation field (essentially a vector field) to control the traveling paths of groups of pedestrians, which has achieved better control of crowds. We further observe that the paths of the individual pedestrians can be considered as

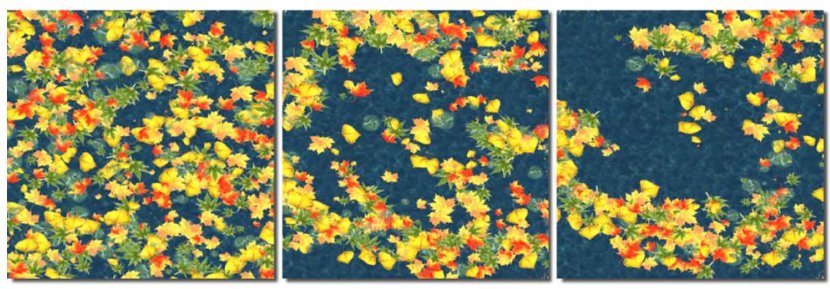

Fig. 14. An animation of fallen leaves advected by a time-varying flow. The leaves are self-spinning according to the advection flow and their orientation direction. This scene contains 1,000 particles. Please see the accompanying video for the spinning effect, available in the online supplemental material.

pathlines, thus can be designed and controlled using our system. In our steerable crowd simulation, the crowds are driven by both a gradient field $G$ derived from the cost function introduced in the continuum crowd technique [39] and a designed time-varying field $F$. The final direction that each pedestrian will take is the weighted sum of these two fields $\omega_{G} G+\omega_{F} F$. Different combinations of weights will determine how closely the crowd follows the specified paths. In the example shown in Fig. 15, we compare the results of different combinations: $\omega_{G}=0.4, \omega_{F}=0.6$ (top) and $\omega_{G}=$ $0.53, \omega_{F}=0.47$ (bottom). The swirling pattern of the paths was created to show the difference between pathlines and streamlines. Streamlines cannot achieve such self-intersecting patterns.

\subsection{Artistic Painterly Animation}

In painterly rendering, the brush stroke orientations are typically guided by a vector field [16], [14], [51]. A timevarying vector field can also be applied to a static image to achieve animating effect in certain regions, such as background, to make the static photo seem alive [5]. Fig. 1 provides such an example. The effect of the evolution of one vortex is inserted to the lower part of the painting to provide water animation. The input time-varying vector field is used to orient the brush strokes as well as advecting them along the flow directions. Fig. 16 shows another

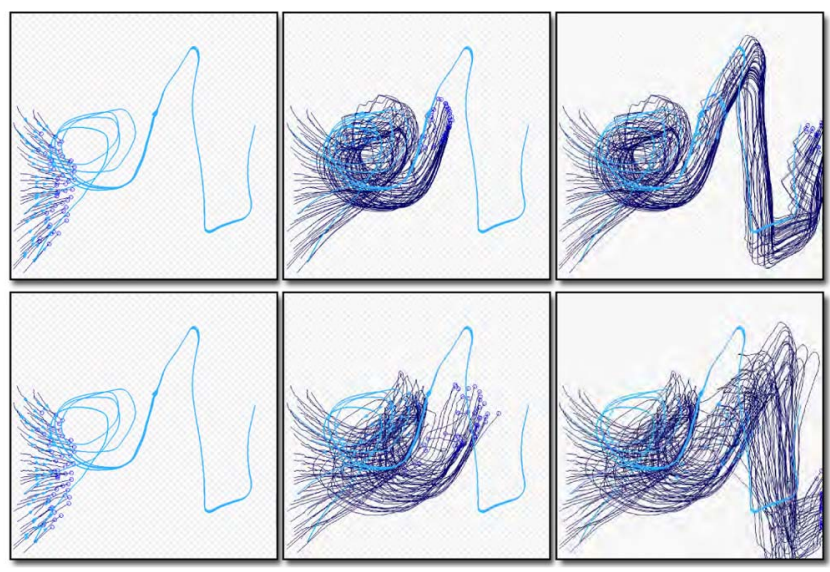

Fig. 15. This example demonstrates a crowd simulation driven by the combination of a social force $G$ with a designed time-varying vector field $F$. The top shows the results of the combination $0.4 G+0.6 F$, and the bottom is $0.53 G+0.47 F$. The cyan curves are the reference pathlines based on the initial positions of the pedestrians and the underlying timevarying flow. The brown curves are the actual paths that the pedestrians have taken. 

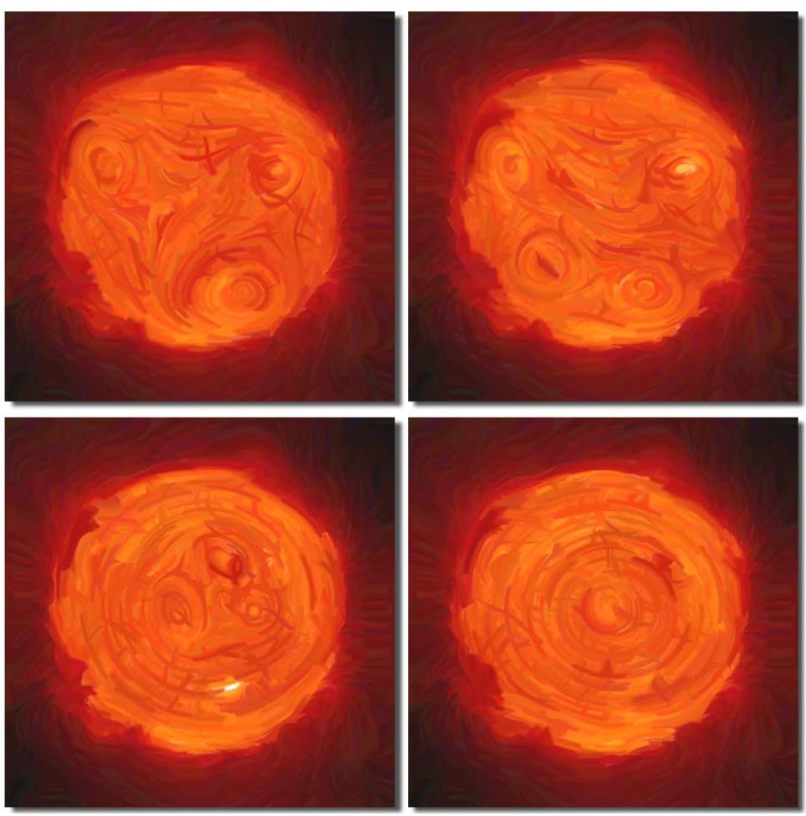

Fig. 16. The effect of a burning sun.

example where several vortices are inserted to provide a burning effect to the original steady image. These vortices interact with each other and eventually collapse into a large vortex in the center. Both fields of these two examples were created using key-frame design, although they could also be generated using element-based design.

\subsection{Performance}

For all the examples shown in this paper, the initialization of a planar time-varying field with 100 frames defined on a $65 \times 65$ regular grid typically takes less than 5 seconds on a $3 \mathrm{GHz}$ PC with $4 \mathrm{~GB}$ RAM. For the design on surfaces (up to 20,000 vertices), it can take up to 3.5 minutes to generate the field with 100 frames without optimization and with an error threshold of $1 . e-10$ and maximum iteration number of 400 for the bi-Conjugate Gradient solver. Note that a direct solver could be applied, such as the Cholesky decomposition. However, when a time-varying vector field with a long sequence is created on a large mesh, the memory usage may not be efficient for such a direct decomposition method.

\subsection{Evaluation and Discussion}

To evaluate the generated time-varying vector fields, we display a plot showing the change of the instantaneous field over time for each example field used in the paper (Fig. 17). This plot allows us to visualize the temporal coherence of a time-varying vector field. The $X$ axis of each plot is the frame index and the $Y$ axis is the total change of the vector field computed as $y\left(x_{i}\right)=\sum_{k}\left\|V\left(\mathbf{v}_{\mathbf{k}} ; t_{i+1}\right)-V\left(\mathbf{v}_{\mathbf{k}} ; t_{i}\right)\right\|$ where $V\left(\mathbf{v}_{\mathbf{k}} ; t_{i}\right)$ is the vector value at vertex $k$ at time $t_{i}$. According to our smoothness assumption in the introduction, the smaller the $y\left(x_{i}\right)$, the slower and smoother the change is. From the plots, we can see that the fields generated using the key-frame design combined with the spatial-temporal Laplacian are typically smooth because of its energy minimization nature. The element-based method could generate fields with larger fluctuation due to the occurrence of unexpected features or insufficient sampling along an
Element-based Design

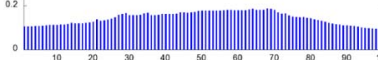

Fig.8: Combined basis field example

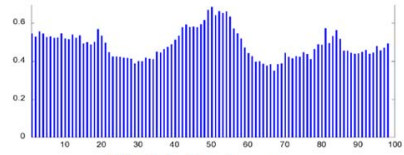

Fig.14: For leaves

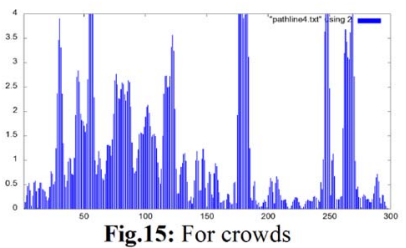

Field deformation

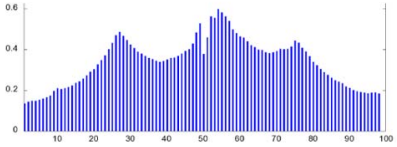

Fig.10: For happy Buddha

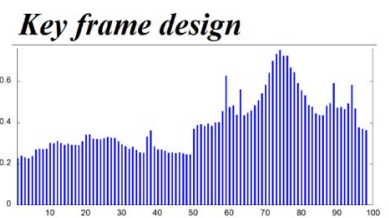

Fig.1: For water effect in a photo 0.2

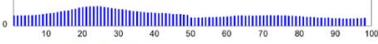

Fig.9: For moving stormts

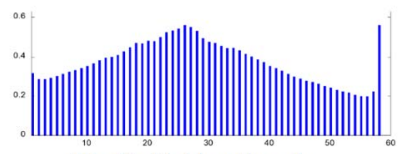

Fig.12: Fluid on the sphere

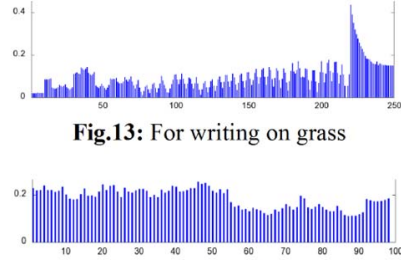

Fig.16: For burning sun
Fig. 17. Coherence plots of the time-varying vector fields used in the paper.

integral curve (e.g., the pathline design shown in Fig. 15). Field deformation also generates fields with large variations over time. This is because of the large change of the transformation matrix or the reference streamline in succeeding times. However, this issue is not fundamental and can be resolved by simply increasing the time sampling to capture the smooth transition of the features that are manipulated. In terms of which design scenario should be used for a given situation, it is application dependent. It is determined by what and how the graphics properties need to be controlled in the specific applications, as demonstrated through various applications in this section. For instance, if the path of certain local graphics primitives (e.g., a vortex in Fig. 5 and the path of a group of pedestrian in Fig. 15) need to be controlled exactly, the element-based design can be employed. If the exact states at some desired times have to be met (e.g., the writing on the grass in Fig. 13), the key-frame design is more suitable. In addition, element-based design and the field deformation approach may provide full control of the local behaviors of the flow at and near the prescribed elements and the representative streamline, but could be labor intensive if the number of local patterns that need to be controlled is large. Key-frame design is effective if instantaneous appearance is the main goal and only a few instantaneous fields at the desired times are required to meet, but it lacks the control of the rest of the field. An ideal solution would be the combination of these different approaches to devise a more flexible and thorough design framework. We plan to investigate this in the future work.

\section{Conclusion and Future Work}

This paper addresses the problem of the design of timevarying vector fields on $2 \mathrm{D}$ domains. We have identified a set of design requirements for different applications as well as two different uses of time-varying vector fields, i.e., for orienting graphical primitives or advecting objects. A 
number of design scenarios and the corresponding design metaphors are then discussed based on these requirements for different purposes in computer graphics. Efficient algorithms are introduced to generate time-varying vector fields from the user-specified design metaphors. A number of editing operations with certain topological guarantees are introduced to enable the fine adjustment of the obtained fields. We have incorporated the present techniques into a design system for the modeling of various time-varying vector fields. To our knowledge, the presented design system is the first of its kind for general time-varying vector field design with bifurcation control. This work opens a new direction in the area of field design which can be extended to the more complex time-varying field design problems. For instance, the same framework can be easily modified to handle the design of time-varying tensor fields by extending the time-varying singular elements to the elements for degenerate points in the element-based design or solving a tensor-based spatial-temporal Laplacian in the key-frame design.

There are a number of future research directions. First, the present generation techniques do not guarantee the desired topology over time, especially for key-frame design. Only the topology at the key-frame fields are defined. More comprehensive control of topology in between key-frame fields is needed. Second, the bifurcation design is an important component in time-varying vector field design as shown in the paper. More flexible and sophisticated design techniques for bifurcations are much desired. We also wish to extend our system to handle a variety of bifurcations that may involve more sophisticated features such as periodic orbits and separation and attachment lines. Third, we plan to explore other flow descriptors including streaklines, timelines [7], and Lagrangian coherent structures [12]. Fourth, more comprehensive combinations of different design functionality and generation techniques, such as combining the presented design with physically based simulation, should be studied to support more complex design tasks in the future. Finally, extending the design techniques for $2 \mathrm{D}$ fields to $3 \mathrm{D}$ ones will be more challenging yet important for computer graphics.

\section{ACKNOWLEDGMENTS}

We would like to thank Dr. Konstantin Mischaikow for the valuable discussion on the topology and dynamics of vector fields, which initiated this work. We also thank Dr. Mark van Langeveld for the valuable discussion on potential applications. We appreciate the help by Timothy O'Keefe on proofreading the paper. This work was supported by NSF IIS-0546881, IIS-0917308, OCI-0906379, and CCF0830808 award. Guoning Chen was partially supported by King Abdullah University of Science and Technology (KAUST) Award No. KUS-C1-016-04 and DOE VACET.

\section{REFERENCES}

[1] P. Alliez, D. Cohen-Steiner, O. Devillers, B. Lévy, and M. Desbrun, "Anisotropic Polygonal Remeshing," ACM Trans. Graphics, vol. 22, no. 3, pp. 485-493, 2003.

[2] A.W. Bargteil, F. Sin, J.E. Michaels, T.G. Goktekin, and J.F. O’Brien, "A Texture Synthesis Method for Liquid Animations," Proc ACM SIGGRAPH/Eurographics Symp. Computer Animation (SCA '06), pp. 345-351, Sept. 2006.
[3] G. Chen, K. Mischaikow, R.S. Laramee, P. Pilarczyk, and E. Zhang, "Vector Field Editing and Periodic Orbit Extraction Using Morse Decomposition," IEEE Trans. Visualization and Computer Graphics, vol. 13, no. 4, pp. 769-785, July-Aug. 2007.

[4] S. Chenney, "Flow Tiles," Proc. ACM SIGGRAPH/Eurographics Symp. Computer Animation (SCA '04), pp. 233-242, 2004.

[5] Y.-Y. Chuang, D.B. Goldman, K.C. Zheng, B. Curless, D.H. Salesin, and R. Szeliski, "Animating Pictures with Stochastic Motion Textures," ACM Trans. Graphics, vol. 24, no. 3, pp. 853-860, 2005.

[6] K. Crane, M. Desbrun, and P. Schröder, "Trivial Connections on Discrete Surfaces," Computer Graphics Forum (SGP), vol. 29, no. 5, pp. 1525-1533, 2010.

[7] T. Faber, Fluid Dynamics for Physicists. Cambridge Univ. Press, 1995.

[8] M. Fisher, P. Schröder, M. Desbrun, and H. Hoppe, "Design of Tangent Vector Fields," ACM Trans. Graphics, vol. 26, no. 3, pp. 56:1-56:9, 2007.

[9] M.S. Floater, "Mean Value Coordinates," Computer Aided Geometric Design, vol. 20, no. 1, pp. 19-27, 2003.

[10] H. Fu, Y. Wei, C.-L. Tai, and L. Quan, "Sketching Hairstyles," Proc. Fourth Eurographics Workshop Sketch-based Interfaces and Modeling (SBIM '07), pp. 31-36, 2007.

[11] J. Hale and H. Kocak, Dynamics and Bifurcations. Springer, 1991.

[12] G. Haller, "Finding Finite-Time Invariant Manifolds in TwoDimensional Velocity Fields," Chaos, vol. 10, no. 1, pp. 99-108, 2000

[13] J. Han, K. Zhou, L.-Y. Wei, M. Gong, H. Bao, X. Zhang, and B. Guo, "Fast Example-Based Surface Texture Synthesis via Discrete Optimization," The Visual Computer, vol. 22, no. 9, pp. 918-925, 2006.

[14] J. Hays and I. Essa, "Image and Video Based Painterly Animation," Proc. Third Int'l Symp. Non-Photorealistic Animation and Rendering (NPAR '04), pp. 113-120, 2004.

[15] J.L. Helman and L. Hesselink, "Representation and Display of Vector Field Topology in Fluid Flow Data Sets," Computer, vol. 22, no. 8, pp. 27-36, Aug. 1989.

[16] A. Hertzmann, "Painterly Rendering with Curved Brush Strokes of Multiple Sizes," Proc. SIGGRAPH'98, pp. 453-460, 1998.

[17] A. Hertzmann and K. Perlin, "Painterly Rendering for Video and Interaction," Proc. First Int'l Symp. Non-Photorealistic Animation and Rendering (NPAR '00), pp. 7-12, 2000.

[18] M. Kagaya, W. Brendel, Q. Deng, T. Kesterson, S. Todorovic, P.J. Neill, and E. Zhang, "Video Painting with Space-Time-Varying Style Parameters," IEEE Trans. Visualization and Computer Graphics, vol. 17, no. 1, pp. 74-87, Jan. 2011.

[19] V. Kwatra, D. Adalsteinsson, T. Kim, N. Kwatra, M. Carlson, and M. Lin, "Texturing Fluids," IEEE Trans. Visualization and Computer Graphics, vol. 13, no. 5, pp. 939-952, Sept.-Oct. 2007.

[20] V. Kwatra, I. Essa, A. Bobick, and N. Kwatra, "Texture Optimization for Example-Based Synthesis," ACM Trans. Graphics, vol. 24, pp. 795-802, Aug. 2005.

[21] Y.-K. Lai, M. Jin, X. Xie, Y. He, J. Palacios, E. Zhang, S.-M. Hu, and X. Gu, "Metric-Driven Rosy Field Design and Remeshing," IEEE Trans. Visualization and Computer Graphics, vol. 16, no. 1, pp. 95108, Jan.-Feb. 2010.

[22] R.S. Laramee, B. Jobard, and H. Hauser, "Image Space Based Visualization of Unsteady Flow on Surfaces," Proc. IEEE 14th Visualization (VIS '03), pp. 131-138, Oct. 2003.

[23] S. Lefebvre and H. Hoppe, "Appearance-Space Texture Synthesis," ACM Trans. Graphics, vol. 25, no. 3, pp. 541-548, 2006.

[24] C. Ma, L.-Y. Wei, B. Guo, and K. Zhou, "Motion Field Texture Synthesis," ACM Trans. Graphics, vol. 28, no. 5, pp. 110:1-110:8, 2009.

[25] O. Mallo, R. Peikert, C. Sigg, and F. Sadlo, "Illuminated Lines Revisited," Proc. IEEE Visualization (VIS '05), pp. 19-26, 2005.

[26] F. Neyret, "Advected Textures," Proc. ACM SIGGRAPH/Eurographics Symp. Computer Animation (SCA '03), pp. 147-153, 2003.

[27] J. Palacios and E. Zhang, "Rotational Symmetry Field Design on Surfaces," ACM Trans. Graphics, vol. 26, no. 3, pp. 56:1-56:10, 2007.

[28] R. Parent, Computer Animation: Algorithms and Techniques, second ed. Morgan Kaufmann Publishers, Inc., 2007.

[29] S. Patil, J. van den Berg, S. Curtis, M.C. Lin, and D. Manocha, "Directing Crowd Simulations Using Navigation Fields," IEEE Trans. Visualization and Computer Graphics, vol. 17, no. 2, pp. 244254, Feb. 2011.

[30] F. Pighin, J.M. Cohen, and M. Shah, "Modeling and Editing Flows Using Advected Radial Basis Functions," Proc. ACM SIGGRAPH/ Eurographics Symp. Computer Animation (SCA '04), pp. 223-232, 2004. 
[31] E. Praun, F. Adam, and H. Hugues, "Lapped Textures," Proc. SIGGRAPH '00, pp. 465-470, 2000.

[32] W.H. Press, S.A. Teukolsky, W.T. Vetterling, and B.P. Flannery, Numerical Recipes in C: The Art of Scientific Computing. Cambridge Univ. Press, 1992.

[33] N. Ray, W.C. Li, B. Lévy, and A.S. an d Pierre Alliez, "Periodic Global Parameterization," ACM Trans. Graphics, vol. 25, no. 4, pp. 1460-1485, 2006.

[34] N. Ray, B. Vallet, W.-C. Li, and B. Levy, "N-Symmetry Direction Field Design," ACM Trans. Graphics, vol. 27, no. 2, pp. 10:1-10:13, 2008.

[35] J. Stam, "Stable Fluids," Proc. SIGGRAPH '99, pp. 121-128, 1999.

[36] J. Stam, "Flows on Surfaces of Arbitrary Topology," ACM Trans. Graphics, vol. 22, no. 3, pp. 724-731, July 2003.

[37] H. Theisel, "Designing 2D Vector Fields of Arbitrary Topology," Proc. Eurographics Conf., vol. 21, no. 3, pp. 595-604, July 2002.

[38] H. Theisel, T. Weinkauf, H.-C. Hege, and H.-P. Seidel, "Topological Methods for 2D Time-Dependent Vector Fields Based on Stream Lines and Path Lines," IEEE Trans. Visualization and Computer Graphics, vol. 11, no. 4, pp. 383-394, July-Aug. 2005.

[39] A. Treuille, S. Cooper, and Z. Popović, "Continuum Crowds," ACM Trans. Graphics, vol. 25, no. 3, pp. 1160-1168, 2006.

[40] X. Tricoche, G. Scheuermann, and H. Hagen, "Topology-based Visualization of Time-Dependent 2D Vector Fields," Proc. Data Visualization, pp. 117-126, 2001.

[41] G. Turk, "Texture Synthesis on Surfaces," Proc. SIGGRAPH '01, pp. 347-354, 2001.

[42] G. Turk and J.F. O' brien, "Modelling with Implicit Surfaces That Interpolate," ACM Trans. Graphics, vol. 21, no. 4, pp. 855-873, 2002.

[43] J. van Wijk, "Image Based Flow Visualization for Curved Surfaces," Proc. IEEE 14th Visualization (VIS '03), pp. 123-130, 2003.

[44] J.J. van Wijk, "Image Based Flow Visualization," ACM Trans. Graphics, vol. 21, no. 3, pp. 745-754, July 2002.

[45] W. von Funck, H. Theisel, and H.-P. Seidel, "Vector Field Based Shape Deformations," ACM Trans. Graphics, vol. 25, no. 3, pp. 1118-1125, 2006.

[46] L.Y. Wei and M. Levoy, "Texture Synthesis over Arbitrary Manifold Surfaces," Proc. SIGGRAPH '01, pp. 355-360, 2001.

[47] J. Wejchert and D. Haumann, "Animation Aerodynamics," Proc. SIGGRAPH '91, pp. 19-22, 1991.

[48] K. Xu, D. Cohne-Or, T. Ju, L. Liu, H. Zhang, S. Zhou, and Y. Xiong, "Feature-Aligned Shape Texturing," ACM Trans. Graphics, vol. 28, no. 5, pp. 108:1-108:7, 2009

[49] K. Xu, H. Zhang, D. Cohen-Or, and Y. Xiong, "Dynamic Harmonic Fields for Surface Processing," Computers and Graphics, vol. 33, no. 3, pp. 391-398, 2009.

[50] L. Xu, J. Chen, and J. Jia, "A Segmentation Based Variational Model for Accurate Optical Flow Estimation," Proc. 10th European Conf. Computer Vision (ECCV'08), pp. 671-684, 2008.

[51] E. Zhang, K. Mischaikow, and G. Turk, "Vector Field Design on Surfaces," ACM Trans. Graphics, vol. 25, no. 4, pp. 1294-1326, 2006.

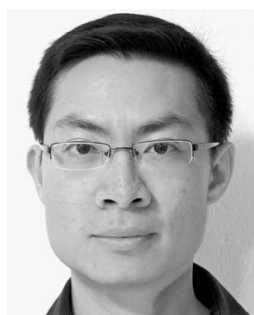

Guoning Chen received the bachelors degree in 1999 from Xi'an Jiaotong University, China and the masters degree in 2002 from Guangxi University, China. In 2009, he received the PhD degree in computer science from Oregon State University. His research interests include scientific visualization, computational topology, and computer graphics. Currently, he is a postdoctoral research associate in Scientific Computing and Imaging $(\mathrm{SCl})$ Institute at the University of Utah. $\mathrm{He}$ is a member of the IEEE.

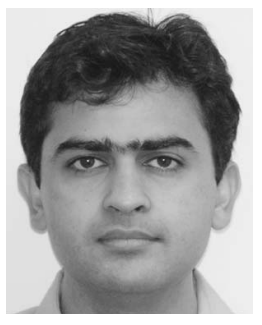

Vivek Kwatra received the BTech degree in computer science and engineering from the Indian Institute of Technology (IIT) Delhi, India, in 1999 and the MS and $\mathrm{PhD}$ degrees in computer science from the Georgia Institute of Technology in 2004 and 2005, respectively. He was a postdoctoral researcher in the Computer Science Department at the University of North Carolina, Chapel Hill, from 2005 to 2007 . He is currently working at Google as a research scientist.

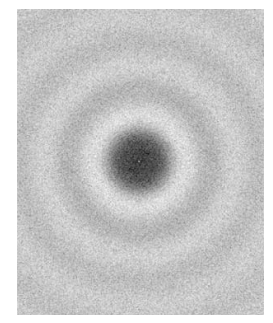

Li-Yi Wei received the $\mathrm{PhD}$ degree from Stanford in 2001. He is an associate professor at The University of Hong Kong. He has been with Microsoft Research from 2005 to 2011 and NVIDIA from 2001 to 2005.

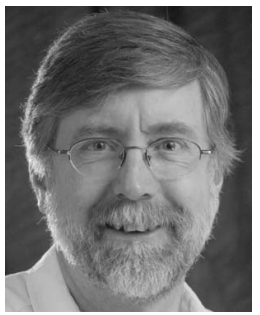

Charles D. Hansen received the BS degree in computer science from Memphis State University in 1981 and the $\mathrm{PhD}$ degree in computer science from the University of Utah in 1987 . He is a professor of computer science at the University of Utah and an associate director of the SCI Institute. From 1989 to 1997, he was a technical staff member in the Advanced Computing Laboratory $(\mathrm{ACL})$ located at Los Alamos National Laboratory, where he formed and directed the visualization efforts in the ACL. He was a Bourse de Chateaubriand postdoc fellow at INRIA, Rocquencourt France, in 1987 and 1988. His research interests include large-scale scientific visualization and computer graphics. He is a fellow of the IEEE.

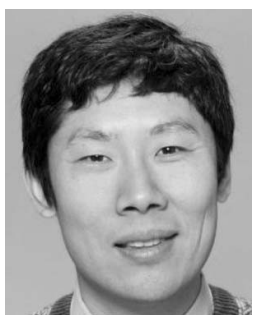

Eugene Zhang received the $\mathrm{PhD}$ degree in computer science in 2004 from Georgia Institute of Technology. He is currently an associate professor at Oregon State University, where he is a member of the School of Electrical Engineering and Computer Science. His research interests include computer graphics, scientific visualization, geometric modeling, and computational topology. He received an National Science Foundation (NSF) CAREER award in 2006. He is a senior member of the IEEE and the ACM.

For more information on this or any other computing topic, please visit our Digital Library at www.computer.org/publications/dlib. 